\title{
Upper bound on three-tangles of reduced states of four-qubit pure states
}

\author{
S. Shelly Sharma, ${ }^{1,}$ and N. K. Sharma 2 , \\ ${ }^{1}$ Departamento de Fisica, Universidade Estadual de Londrina, Londrina-PR, Brazil \\ ${ }^{2}$ Departamento de Matematica, Universidade Estadual de Londrina, Londrina 86051-990, PR Brazil
}

\begin{abstract}
Closed formulae for upper bound on three-tangles of three-qubit reduced states in terms of threequbit invariant polynomials of pure four-qubit states are obtained. Our results offer tighter constraints on total three-way entanglement of a given qubit with the rest of the system than those used in ref. [28, 29] to verify monogamy of four-qubit quantum entanglement.
\end{abstract}

Keywords: Multipartite entanglement, Three-tangle, Four-qubit pure states, Three-qubit invariants of pure state, Local unitary invariance

\section{INTRODUCTION}

Statistical mixtures of quantum states have quantum as well classical correlations. Detecting and quantifying nonclassicality (or quantumness) of multipartite quantum system is of special interest in quantum optics [1 $[3$ ], quantum information [4], and even in quantum biology [5, 6]. In particular, detecting and quantifying quantum entanglement is increasingly important to experiments in quantum information science [7 9 ] and it also plays a crucial role in investigations in quantum statistical physics, e.g., in studying phase transitions [10, 11]. State coefficients of a pure quantum state contain complete information about the correlations amongst the subsystems. Local unitary invariance constraints allow us to construct unitary invariant functions of state coefficients to quantify two-way, three-way, four-way,..., $\mathrm{N}$-way entanglement of an $\mathrm{N}$-qubit state. Global negativity [12, 13] or equivalently concurrence 14] of a pure state of two qubits quantifies two-way correlations. Three tangle [15] is an entanglement monotone that quantifies three-way entanglement of a three-qubit state. Four-way entanglement of a four-qubit state is quantified by four-tangle [16], which for pure state is a degree eight function of state coefficients. Three-tangle and four-tangle for pure quantum states are moduli of specific local special unitary (LSUT) invariants, that are polynomial functions of state coefficients. Non-locality properties of an entangled state do not change under unitary operations acting independently on each of its sub-systems. The idea of describing entanglement by means of local-unitary invariants has been explored in refs. [17-19]. It is shown in ref. [19] that given a basis of stochastic local operations and classical communication (SLOCC) covariants of degree $d$ in variables, the scalar products with respect to auxilliary variables, form a basis of the space of local unitary (LUT) invariants. Squared modulus $|I|^{2}$ of a SLOCC invariant is a LUT and LSUT invariant.

Using an intuitive approach coupled to classical theory of invariants it has been shown in ref. [20] that one can construct local unitary invariants characterizing a pure state of $\mathrm{N}$ two-level quantum systems (qubits) in terms of N-1 qubit invariants. A natural question is, can we write entanglement of (N-1)-qubit marginals of an N-qubit pure state in terms of invariants of the N-qubit pure state? In this letter, we focus on four qubit states and their three-qubit marginals. Entanglement measures for reduced states are, generally, based on the convex roof of a quantity defined on pure states. In most of the cases they are not computable as there are no efficient ways to calculate convex roofs. Although a closed formula for the three-tangle [15] of pure states is known, for mixed states no such formula is available. Solutions to finding the convex roof of three-tangle for special families of states are, however, available in 21 24]. A method to compute entanglement measures based on convex roof constructions is also given in ref. [25]. Recently, Osterloh [26], obtained three tangles of nine classes of four qubit states [27], by finding a decomposition of three qubit mixed state such that its entanglement lies on minimal characteristic curve. We outline the formalism to construct three-tangle of reduced state from relevant three-qubit polynomial invariants of the four qubit pure state. Local unitary invariance of entanglement is the basic principle underlying the construction. In the most general case, it involves finding the roots of a quartic equation. The roots may be found analytically or numerically. Closed formulae for upper bound on three tangles of nine classes of four qubit states 27], are obtained and the results compared with those of ref. [28, 29]. Our results offer tighter constraints on three-way entanglement of a given qubit with the rest of the system than those used in ref. [28, 29].

\footnotetext{
*Electronic address: shelly@uel.br

†Electronic address: nsharma@uel.br
} 
We start by defining three tangle on pure and mixed states in section II, outline the formalism in sections III and IV, discuss the upper bounds on three tangles for nine classes of four-qubit states in section $\mathrm{V}$ and optimal decomposition of a rank two mixed state in section VI. Section VII contains the concluding remarks.

\section{DEFINITION OF THREE TANGLE}

Consider a three-qubit pure state

$$
\left|\Psi_{3}\right\rangle=\sum_{i_{1}, i_{2}, i_{3}} a_{i_{1} i_{2} i_{3}}\left|i_{1} i_{2} i_{3}\right\rangle, \quad i_{m}=0,1
$$

where state coefficients $a_{i_{1} i_{2} i_{3}}$ are complex numbers and $i_{m}$ refers to computational basis state of qubit $A_{m}$, $(m=1,2,3)$. Let qubit $A_{1}$ be the focus qubit. Using the notation from ref. [20], we define $D_{\left(A_{3}\right)_{i_{3}}}^{00}=$ $a_{00 i_{3}} a_{11 i_{3}}-a_{10 i_{3}} a_{01 i_{3}},\left(i_{3}=0,1\right)$ as determinant of a two-way negativity font and $D^{00 i_{3}}=a_{00 i_{3}} a_{11 i_{3}+1}-a_{10 i_{3}} a_{01 i_{2}}+1$, $\left(i_{3}=0,1\right)$ is determinant of a three-way negativity font. Three tangle of pure state $\left|\Psi_{3}\right\rangle$ as defined in ref. [15] is equal to the modulus of a polynomial invariant of degree four that is $\tau_{A_{1}\left|A_{2}\right| A_{3}}\left(\left|\Psi_{3}\right\rangle\right)=4\left|I_{3,4}\right|$, where

$$
I_{3,4}=\left(D^{000}+D^{001}\right)^{2}-4 D_{\left(A_{3}\right)_{0}}^{00} D_{\left(A_{3}\right)_{1}}^{00} .
$$

The entanglement measure $\tau_{A_{1}\left|A_{2}\right| A_{3}}\left(\left|\Psi_{3}\right\rangle\right)$ is extended to a mixed state of three qubits via convex roof extension that is

$$
\left[\tau_{A_{1}\left|A_{2}\right| A_{3}}\left(\rho_{3}\right)\right]^{\frac{1}{2}}=\min _{\left\{p_{i},\left|\phi^{(i)}\right\rangle\right\}} \sum_{i} p_{i}\left[\tau_{A_{1}\left|A_{2}\right| A_{3}}\left(\left|\phi^{(i)}\right\rangle\right)\right]^{\frac{1}{2}}
$$

where minimization is taken over all possible decompositions $\left\{p_{i},\left|\phi^{(i)}\right\rangle\right\}$ of $\rho_{3}$.

\section{UPPER BOUND ON THREE TANGLE OF A RANK TWO REDUCED STATE}

Purification of a rank two three-qubit mixed state is a four-qubit pure state. In this section, we describe the construction of upper bound on three tangle of marginal state of a four qubit pure state in terms of three-qubit and four-qubit unitary invariants of the pure state. In the most general case, it involves finding the roots of a quartic equation. The roots may be found analytically or numerically. Analytical form of relevant three-qubit and four-qubit invariants is to be found in Sec. V of ref. [20]. Upper bounds on three-qubit mixed state entanglement, used in ref. [28, 29] in the context of a monogamy of quantum entanglement, had been calculated by using an algorithm [30] to construct the entanglement-minimizing decomposition for $\rho$. Our results offer tighter constraints on three-way entanglement of a given qubit with the rest of the system than those used in ref. [28, 29].

Consider a general four qubit pure state, written as

$$
|\Psi\rangle=\sum_{i_{1}, i_{2}, i_{3}}\left(a_{i_{1} i_{2} i_{3} 0}\left|i_{1} i_{2} i_{3} 0\right\rangle+a_{i_{1} i_{2} i_{3} 1}\left|i_{1} i_{2} i_{3} 1\right\rangle\right), \quad\left(i_{m}=0,1\right)
$$

where qubits are in the order $\left(A_{1}, A_{2}, A_{3}, A_{4}\right)$ corresponding to the state $\left|i_{1} i_{2} i_{3} i_{4}\right\rangle$. Choosing qubit $A_{1}$ as the focus qubit, we identify $D_{\left(A_{3}\right)_{i_{3}}\left(A_{4}\right)_{i_{4}}}^{00}=a_{00 i_{3} i_{4}} a_{11 i_{3} i_{4}}-a_{10 i_{3} i_{4}} a_{01 i_{3} i_{4}}$ (two-way), $D_{\left(A_{2}\right)_{i_{2}}\left(A_{4}\right)_{i_{4}}}^{00}=a_{0 i_{2} 0 i_{4}} a_{1 i_{2} 1 i_{4}}-a_{1 i_{2} 0 i_{4}} a_{0 i_{2} 1 i_{4}}$ (two-way), $D_{\left(A_{2}\right)_{i_{2}}\left(A_{3}\right)_{i_{3}}}^{00}=a_{0 i_{2} i_{3} 0} a_{1 i_{2} i_{3} 1}-a_{1 i_{2} i_{3} 0} a_{0 i_{2} i_{3} 1}$ (two-way), $D_{\left(A_{4}\right)_{i_{4}}}^{00 i_{3}}=a_{00 i_{3} i_{4}} a_{11, i_{3} \oplus 1, i_{4}}-a_{10 i_{3} i_{4}} a_{01, i_{3} \oplus 1, i_{4}}($ threeway), $D_{\left(A_{3}\right)_{i_{3}}}^{00 i_{4}}=a_{00 i_{3} i_{4}} a_{11 i_{3}, i_{4} \oplus 1}-a_{10 i_{3} i_{4}} a_{01 i_{3}, i_{4} \oplus 1}$ (three-way), $D_{\left(A_{2}\right)_{i_{2}}}^{00 i_{4}}=a_{0 i_{2} 0 i_{4}} a_{1 i_{2} 1 i_{4} \oplus 1}-a_{1 i_{2} 0 i_{4}} a_{0 i_{2} 1 i_{4} \oplus 1}($ three-way), and $D^{00 i_{3} i_{4}}=a_{00 i_{3} i_{4}} a_{11, i_{3} \oplus 1, i_{4} \oplus 1}-a_{10 i_{3} i_{4}} a_{01, i_{3} \oplus 1, i_{4} \oplus 1^{-}}$(four-way) as the determinants of negativity fonts. We shall also use the three and four qubit invariants constructed in section $\mathrm{V}$ of ref. [20]. Three-qubit invariants of interest for the triple $A_{1} A_{2} A_{3}$ in state $|\Psi\rangle$ comprise a set denoted by $\left\{\left(I_{3,4}\right)_{A_{4}}^{4-m, m}: m=0\right.$ to 4$\}$. The form of elements of the set in terms of determinants of negativity fonts is given in Appendix A The elements in set $\left\{\left(I_{3,4}\right)_{A_{4}}^{4-m, m}: m=0\right.$ to 4$\}$ are invariant with respect to local unitaries on qubits $A_{1}, A_{2}$, and $A_{3}$. Four-qubit invariant that quantifies the sum of three-way and four-way correlations [20] of triple $A_{1} A_{2} A_{3}$, reads as

$$
N_{4,8}^{A_{1} A_{2} A_{3}}=6\left|\left(I_{3,4}\right)_{A_{4}}^{2,2}\right|^{2}+4\left|\left(I_{3,4}\right)_{A_{4}}^{3,1}\right|^{2}+4\left|\left(I_{3,4}\right)_{A_{4}}^{1,3}\right|^{2}+\left|\left(I_{3,4}\right)_{A_{4}}^{4,0}\right|^{2}+\left|\left(I_{3,4}\right)_{A_{4}}^{0,4}\right|^{2}
$$


while degree eight invariant that detects genuine four-body entanglement of a four-qubit state is given by

$$
I_{4,8}=3\left(\left(I_{3,4}\right)_{A_{4}}^{2,2}\right)^{2}-4\left(I_{3,4}\right)_{A_{4}}^{3,1}\left(I_{3,4}\right)_{A_{4}}^{1,3}+\left(I_{3,4}\right)_{A_{4}}^{4,0}\left(I_{3,4}\right)_{A_{4}}^{0,4}
$$

Invariant $I_{4,8}$ is written as a function of $A_{1} A_{2} A_{3}$ invariants in Eq. (6). Being independent of the choice of focus qubit, $I_{4,8}$ can be written in alternate forms. Four-tangle based on degree eight invariant is defined [16, 20] as

$$
\tau_{4,8}=16\left|12\left(I_{4,8}\right)\right|
$$

$\operatorname{Set}\left\{\left(I_{3,4}\right)_{A_{3}}^{4-m, m}: m=0\right.$ to 4$\}$ for the triple $A_{1} A_{2} A_{4}$ and $\left\{\left(I_{3,4}\right)_{A_{2}}^{4-m, m}: m=0\right.$ to 4$\}$ for qubits $A_{1} A_{3} A_{4}$ can be constructed from two-qubit invariants of properly selected pair of qubits [20]. In the following sections, the subscript 4 in $\left(I_{3,4}\right)_{A_{3}}^{4-m, m}$ has been dropped, that is $\left(I_{3,4}\right)_{A_{3}}^{4-m, m}=\left(I_{3}\right)_{A_{3}}^{4-m, m}$.

\section{A. Unitary on fourth qubit}

To illustrate the method, we focus on three-qubit reduced state $\rho=\sum_{i=0}^{1} p_{A_{4}}^{(i)}\left|\phi_{A_{4}}^{(i)}\right\rangle\left\langle\phi_{A_{4}}^{(i)}\right|$, obtained by tracing out qubit $A_{4}$ from the state $|\Psi\rangle$. Probability of finding qubit $A_{4}$ in state $|i\rangle$ is denoted by $p_{A_{4}}^{(i)}$. A unitary $U(x)=$ $\frac{1}{\sqrt{1+|x|^{2}}}\left[\begin{array}{cc}1 & -x^{*} \\ x & 1\end{array}\right]$, on qubit $A_{4}$ of state $|\Psi\rangle$ results in an $x$ dependent state

$$
|\Psi(x)\rangle=\sum_{i_{4}=0}^{1}\left|\Phi_{A_{4}}^{\left(i_{4}\right)}(x)\right\rangle\left|i_{4}\right\rangle
$$

where $\left|\Phi_{A_{4}}^{\left(i_{4}\right)}(x)\right\rangle=\sum_{i_{1}, i_{2}, i_{3}} a_{i_{1} i_{2} i_{3} i_{4}}(x)\left|i_{1} i_{2} i_{3}\right\rangle$ is a subnormalized state. Reduced state obtained by tracing out qubit $A_{4}$ reads as

$$
\rho(x)=\sum_{i=0}^{1} p_{A_{4}}^{(i)}(x)\left|\phi_{A_{4}}^{(i)}(x)\right\rangle\left\langle\phi_{A_{4}}^{(i)}(x)\right|
$$

where $\left|\phi_{A_{4}}^{\left(i_{4}\right)}(x)\right\rangle=\frac{\left|\Phi_{A_{4}}^{\left(i_{4}\right)}(x)\right\rangle}{\sqrt{p_{A_{4}}^{(i)}(x)}}$, is a normalized state, and $x$ dependent probabilities are defined as

$$
p_{A_{4}}^{(0)}(x)=\frac{p_{A_{4}}^{(0)}+|x|^{2} p_{A_{4}}^{(1)}}{1+|x|^{2}}, \quad p_{A_{4}}^{(1)}(x)=\frac{p_{A_{4}}^{(1)}+|x|^{2} p_{A_{4}}^{(0)}}{1+|x|^{2}} .
$$

One can verify that the $x$ dependent three-tangle

$$
\tau_{A_{1}\left|A_{2}\right| A_{3}}\left(\left|\phi_{A_{4}}^{(0)}(x)\right\rangle\right)=\frac{4}{\left(p_{A_{4}}^{(0)}(x)\right)^{2}}\left|\left(I_{3}\right)_{A_{4}}^{4,0}(x)\right|,
$$

and

$$
\tau_{A_{1}\left|A_{2}\right| A_{3}}\left(\left|\phi_{A_{4}}^{(1)}(x)\right\rangle\right)=\frac{4}{\left(p_{A_{4}}^{(1)}(x)\right)^{2}}\left|\left(I_{3}\right)_{A_{4}}^{0,4}(x)\right| .
$$

Using the definition of three tangle for mixed states (Eq. (3) ), we can write

$$
\left[\tau_{A_{1}\left|A_{2}\right| A_{3}}\left(\rho_{3}\right)\right]^{\frac{1}{2}} \leq 2 \min _{\{x\}}\left(\left|\left(I_{3}\right)_{A_{4}}^{4,0}(x)\right|^{\frac{1}{2}}+\left|\left(I_{3}\right)_{A_{4}}^{0,4}(x)\right|^{\frac{1}{2}}\right) .
$$

Three qubit invariants $\left(I_{3}\right)_{A_{4}}^{4,0}(x)$ and $\left(I_{3}\right)_{A_{4}}^{0,4}(x)$ are related to elements of the set $\left\{\left(I_{3}\right)_{A_{4}}^{4-m, m}: m=0,4\right\}$ through

$$
\begin{aligned}
\left(I_{3}\right)_{A_{4}}^{4,0}(x)= & \frac{1}{\left(1+|x|^{2}\right)^{2}}\left[\left(I_{3}\right)_{A_{4}}^{4,0}-4 x^{*}\left(I_{3}\right)_{A_{4}}^{3,1}\right. \\
& \left.+6\left(x^{*}\right)^{2}\left(I_{3}\right)^{2,2}-4\left(x^{*}\right)^{3}\left(I_{3}\right)_{A_{4}}^{1,3}+\left(x^{*}\right)^{4}\left(I_{3}\right)_{A_{4}}^{0,4}\right],
\end{aligned}
$$


and

$$
\begin{aligned}
\left(I_{3}\right)_{A_{4}}^{0,4}(x)= & \frac{1}{\left(1+|x|^{2}\right)^{2}}\left[\left(I_{3}\right)_{A_{4}}^{0,4}+4 x\left(I_{3}\right)_{A_{4}}^{1,3}\right. \\
& \left.+6 x^{2}\left(I_{3}\right)_{A_{4}}^{2,2}+4 x^{3}\left(I_{3}\right)_{A_{4}}^{3,1}+\left(I_{3}\right)_{A_{4}}^{4,0} x^{4}\right] .
\end{aligned}
$$

To obtain an upper bound on three tangle of mixed state, we find the value of complex parameter $x_{1}$ such that $\left(I_{3}\right)_{A_{4}}^{4,0}\left(x_{1}\right)=0$, and $x_{2}$ such that $\left(I_{3}\right)_{A_{4}}^{0,4}\left(x_{2}\right)=0$. In the most general case, that involves solving a quartic equation in variable $x$, (obtained from Eq. (13) or (14) ). When the state coefficients are known, the resulting quartic may be solved numerically. However, for the representatives of nine classes of four-qubit entanglement [27] analytic solutions are easily found. By definition the three tangle must satisfy Eq. (12), as such three tangle satisfies the inequality

$$
\left[\tau_{A_{1}\left|A_{2}\right| A_{3}}\left(\rho_{3}\right)\right] \leq 4 \min \left(\left|\left(I_{3}\right)_{A_{4}}^{0,4}\left(x_{1}\right)\right|,\left|\left(I_{3}\right)_{A_{4}}^{4,0}\left(x_{2}\right)\right|\right)
$$

giving us an upper bound on three tangle of the mixed state.

\section{B. Unitary on three qubit state}

If normalized three-qubit states $\left|\phi_{A_{4}}^{(0)}\right\rangle$ and $\left|\phi_{A_{4}}^{(1)}\right\rangle$ are orthogonal to each other then a unitary on three qubit state such that

$$
\left|\phi_{A_{4}}^{(0)}(y)\right\rangle=\frac{1}{\sqrt{1+|y|^{2}}}\left(\left|\phi_{A_{4}}^{(0)}\right\rangle+y\left|\phi_{A_{4}}^{(1)}\right\rangle\right)
$$

and

$$
\left|\phi_{A_{4}}^{(1)}(y)\right\rangle=\frac{1}{\sqrt{1+|y|^{2}}}\left(\left|\phi_{A_{4}}^{(1)}\right\rangle-y^{*}\left|\phi_{A_{4}}^{(0)}\right\rangle\right),
$$

results in a $y$ dependent four-qubit state

$$
|\Psi(y)\rangle=\sum_{i_{4}=0}^{1} \sqrt{p_{A_{4}}^{\left(i_{4}\right)}}\left|\phi_{A_{4}}^{\left(i_{4}\right)}(y)\right\rangle\left|i_{4}\right\rangle,
$$

such that three tangle may be defined as

$$
\left[\tau_{A_{1}\left|A_{2}\right| A_{3}}(\rho)\right]^{\frac{1}{2}}=2 \min _{\{y\}}\left(p_{A_{4}}^{(0)}\left|\left(I_{3}\right)_{A_{4}}^{4,0}(y)\right|^{\frac{1}{2}}+p_{A_{4}}^{(1)}\left|\left(I_{3}\right)_{A_{4}}^{0,4}(y)\right|^{\frac{1}{2}}\right) .
$$

Recalling that three-qubit invariants, $\left(I_{3}\right)_{A_{4}}^{k-m, m}$, are degree four functions of state coefficients, in this case

$$
\begin{aligned}
\left(I_{3}\right)_{A_{4}}^{4,0}(y)= & \frac{1}{\left(1+|y|^{2}\right)^{2}}\left(\frac{\left(I_{3}\right)_{A_{4}}^{4,0}}{\left(p_{A_{4}}^{0}\right)^{2}}+4 y \frac{\left(I_{3}\right)_{A_{4}}^{3,1}}{\sqrt{\left(p_{A_{4}}^{0}\right)^{3} p_{A_{4}}^{1}}}\right. \\
& \left.+6 y^{2} \frac{\left(I_{3}\right)_{A_{4}}^{2,2}}{p_{A_{4}}^{0} p_{A_{4}}^{1}}+4 y^{3} \frac{\left(I_{3}\right)_{A_{4}}^{1,3}}{\sqrt{p_{A_{4}}^{0}\left(p_{A_{4}}^{1}\right)^{3}}}+y^{4} \frac{\left(I_{3}\right)_{A_{4}}^{0,4}}{\left(p_{A_{4}}^{1}\right)^{2}}\right)
\end{aligned}
$$

and

$$
\begin{aligned}
\left(I_{3}\right)_{A_{4}}^{0,4}(y)= & \frac{1}{\left(1+|y|^{2}\right)^{2}}\left(\frac{\left(I_{3}\right)_{A_{4}}^{04}}{\left(p_{A_{4}}^{1}\right)^{2}}-4 y^{*} \frac{\left(I_{3}\right)_{A_{4}}^{1,3}}{\sqrt{\left(p_{A_{4}}^{1}\right)^{3} p_{A_{4}}^{0}}}\right. \\
& \left.+6\left(y^{*}\right)^{2} \frac{\left(I_{3}\right)_{A_{4}}^{2,2}}{p_{A_{4}}^{0} p_{A_{4}}^{1}}-4\left(y^{*}\right)^{3} \frac{\left(I_{3}\right)_{A_{4}}^{3,1}}{\sqrt{p_{A_{4}}^{1}\left(p_{A_{4}}^{0}\right)^{3}}}+\left(y^{*}\right)^{4} \frac{\left(I_{3}\right)_{A_{4}}^{4,0}}{\left(p_{A_{4}}^{0}\right)^{2}}\right) .
\end{aligned}
$$


We look for $\rho\left(y_{1}\right)$ with $\left(I_{3}\right)_{A_{4}}^{4,0}\left(y_{1}\right)=0$, and $\rho\left(y_{2}\right)$ such that $\left(I_{3}\right)_{A_{4}}^{0,4}\left(y_{2}\right)=0$. That gives us an upper bound on $\left[\tau_{A_{1}\left|A_{2}\right| A_{3}}(\rho)\right]^{\frac{1}{2}}$ that is

$$
\left[\tau_{A_{1}\left|A_{2}\right| A_{3}}(\rho)\right]^{\frac{1}{2}} \leq \min \left(2 p_{A_{4}}^{0}\left|\left(I_{3}\right)_{A_{4}}^{0,4}\left(y_{1}\right)\right|^{\frac{1}{2}}, 2 p_{A_{4}}^{1}\left|\left(I_{3}\right)_{A_{4}}^{4,0}\left(y_{2}\right)\right|^{\frac{1}{2}}\right)
$$

If for a given state $p_{A_{4}}^{(0)}=p_{A_{4}}^{(1)}$, then the result obtained by a unitary on fourth qubit coincides with that obtained by a unitary on the three-qubit pure states of the decomposition of the mixed state. When $p_{A_{4}}^{(0)} \neq p_{A_{4}}^{(1)}$, then minima found by unitary on fourth qubit and those calculated by unitary on three qubit state must be compared to find the correct bound on three-tangle for the mixed state. Our results complement the upper bounds of ref. [26] which possibly correspond to those found by applying a unitary on a three qubit marginal state (Eq. 22).

\section{THREE TANGLE AND THREE-QUBIT INVARIANTS OF SIX GROUPS OF STATES}

First of all we notice that since the difference $16\left(N_{4,8}^{A_{i} A_{j} A_{k}}-2\left|I_{4,8}\right|\right)$ is a measure of three-way correlations amongst qubits $A_{i}, A_{j}$, and $A_{k}$ in pure state, the three tangle must satisfy the condition $\tau_{A_{i}\left|A_{j}\right| A_{k}}(\rho) \leq 4 \sqrt{N_{4,8}^{A_{i} A_{j} A_{k}}-2\left|I_{4,8}\right|}$. Evaluation of three-qubit invariants $\left\{\left(I_{3}\right)_{A_{q}}^{4-m, m}: m=0\right.$ to 4$\}$, where $A_{q}$ refers to the qubit which is traced out, shows that three-qubit marginals of states representing nine classes of four qubits belong in six groups. We use unitary on fourth qubit to express upper bound on three tangle in terms of three-qubit invariants for the following cases of interest:

(i) For a given triple of qubits $A_{i} A_{j} A_{k}, N_{4,8}^{A_{i} A_{j} A_{k}}=2\left|I_{4,8}\right|$, therefore three tangle, $\tau_{A_{i}\left|A_{j}\right| A_{k}}(\rho)=0$.

(ii) Only $\left(I_{3}\right)_{A_{q}}^{4,0}$ is non-zero, therefore, $\left|I_{4,8}\right|=0$ and $\tau_{A_{i}\left|A_{j}\right| A_{k}}(\rho) \leq 4\left|\left(I_{3}\right)_{A_{q}}^{4,0}\right|$.

(iii) Only $\left(I_{3}\right)_{A_{q}}^{0,4}$ is non-zero, therefore, $\left|I_{4,8}\right|=0$ and $\tau_{A_{i}\left|A_{j}\right| A_{k}}(\rho) \leq 4\left|\left(I_{3}\right)_{A_{q}}^{0,4}\right|$.

(iv) Non zero three-qubit invariants are $\left(I_{3}\right)_{A_{q}}^{4,0}$ and $\left(I_{3}\right)_{A_{q}}^{2,2}$, therefore

$$
\left(I_{3}\right)_{A_{q}}^{4,0}(x)=\frac{1}{\left(1+|x|^{2}\right)^{2}}\left(\left(I_{3}\right)_{A_{q}}^{4,0}+6\left(x^{*}\right)^{2}\left(I_{3}\right)_{A_{q}}^{2,2}\right)
$$

and

$$
\left(I_{3}\right)_{A_{q}}^{0,4}(x)=\frac{x^{2}}{\left(1+|x|^{2}\right)^{2}}\left(6\left(I_{3}\right)_{A_{q}}^{2,2}+x^{2}\left(I_{3}\right)_{A_{q}}^{4,0}\right)
$$

In this case three tangle satisfies the condition

$$
\tau_{A_{i}\left|A_{j}\right| A_{k}}(\rho) \leq 4\left|\left(I_{3}\right)_{A_{4}}^{4,0}\right| \frac{|| 6\left(I_{3}\right)^{2,2}|-|\left(I_{3}\right)_{A_{4}}^{4,0}||}{\left|6\left(I_{3}\right)^{2,2}\right|+\left|\left(I_{3}\right)_{A_{4}}^{4,0}\right|}
$$

(v) Non zero three-qubit invariants are $\left(I_{3}\right)_{A_{q}}^{0,4}$ and $\left(I_{3}\right)_{A_{q}}^{2,2}$, then to obtain three tangle we use the relations

$$
\left(I_{3}\right)_{A_{q}}^{4,0}(x)=\frac{\left(x^{*}\right)^{2}}{\left(1+|x|^{2}\right)^{2}}\left(6\left(I_{3}\right)_{A_{q}}^{2,2}+\left(x^{*}\right)^{2}\left(I_{3}\right)_{A_{q}}^{0,4}\right)
$$

and

$$
\left(I_{3}\right)_{A_{q}}^{0,4}(x)=\frac{1}{\left(1+|x|^{2}\right)^{2}}\left(\left(I_{3}\right)_{A_{q}}^{0,4}+x^{2} 6\left(I_{3}\right)_{A_{q}}^{2,2}\right)
$$


leading to the condition

$$
\tau_{A_{i}\left|A_{j}\right| A_{k}}(\rho) \leq 4\left|\left(I_{3}\right)_{A_{4}}^{0,4}\right| \frac{|| 6\left(I_{3}\right)^{2,2}|-|\left(I_{3}\right)_{A_{4}}^{0,4}||}{\left|6\left(I_{3}\right)^{2,2}\right|+\left|\left(I_{3}\right)_{A_{4}}^{0,4}\right|} .
$$

(vi) The special case where only $\left(I_{3}\right)_{A_{q}}^{0,4}$ and $\left(I_{3}\right)_{A_{q}}^{1,3}$ are non-zero such that

$$
\begin{aligned}
& \left(I_{3}\right)_{A_{q}}^{4,0}(x)=\frac{\left(x^{*}\right)^{3}}{\left(1+|x|^{2}\right)^{2}}\left(x^{*}\left(I_{3}\right)_{A_{q}}^{0,4}-4\left(I_{3}\right)_{A_{q}}^{1,3}\right), \\
& \left(I_{3}\right)_{A_{q}}^{0,4}(x)=\frac{1}{\left(1+|x|^{2}\right)^{2}}\left(\left(I_{3}\right)_{A_{q}}^{0,4}+4 x\left(I_{3}\right)_{A_{q}}^{1,3}\right),
\end{aligned}
$$

therefore three tangle satisfies the inequality

$$
\tau_{A_{i}\left|A_{j}\right| A_{k}}(\rho) \leq \frac{4\left|\left(I_{3}\right)_{A_{q}}^{0,4}\right|^{3}}{\left|4\left(I_{3}\right)_{A_{q}}^{1,3}\right|^{2}+\left|\left(I_{3}\right)_{A_{q}}^{0,4}\right|^{2}}
$$

\section{THREE-TANGLES FOR NINE CLASSES OF FOUR-QUBIT ENTANGLEMENT}

In this section, we use the results from previous section to write down upper bounds on three-tangles for representatives of nine classes of four qubit states. Our results offer tighter constraints on total three-way entanglement of a given qubit with the rest of the system than those used in refs. [28, 29].

\section{A. Class I}

Class one states are represented by

$$
\begin{aligned}
\left|G_{a b c d}^{(1)}\right\rangle= & \frac{a+d}{2}(|0000\rangle+|1111\rangle)+\frac{a-d}{2}(|0011\rangle+|1100\rangle) \\
& +\frac{b+c}{2}(|0101\rangle+|1010\rangle)+\frac{b-c}{2}(|0110\rangle+|1001\rangle) .
\end{aligned}
$$

For any partition $A_{i} A_{j} A_{k},\left(I_{3}\right)_{A_{l}}^{4,0}=\left(I_{3}\right)_{A_{l}}^{0,4},\left(I_{3}\right)_{A_{l}}^{2,2} \neq 0$, while $\left(I_{3}\right)_{A_{l}}^{3,1}=\left(I_{3}\right)_{A_{l}}^{1,3}=0$. As a result, $16 N_{4,8}^{A_{i} A_{j} A_{k}}=2\left|I_{4,8}\right|$, therefore three tangle, $\tau_{A_{i}\left|A_{j}\right| A_{k}}(\rho)=0$.

\section{B. Class II}

For class two states three-tangle for all four three-qubit partitions has the same value. Consider pure state threequbit invariants for partition $A_{1} A_{2} A_{3}$ in representative state

$$
\begin{aligned}
\left|G_{\text {adc }}^{(2)}\right\rangle= & \frac{a+d}{2}(|0000\rangle+|1111\rangle)+\frac{a-d}{2}(|0011\rangle+|1100\rangle) \\
& +c(|0101\rangle+|1010\rangle)+|0110\rangle .
\end{aligned}
$$

Three-qubit invariants have values

$$
\left(I_{3}\right)_{A_{4}}^{4,0}=\frac{c\left(a^{2}-d^{2}\right)}{\left(|a|^{2}+|d|^{2}+2|c|^{2}+1\right)^{2}},\left(I_{3}\right)_{A_{4}}^{2,2}=\frac{\left(a^{2}-c^{2}\right)\left(d^{2}-c^{2}\right)}{6\left(|a|^{2}+|d|^{2}+2|c|^{2}+1\right)^{2}},
$$


while $\left(I_{3}\right)_{A_{4}}^{3,1}=\left(I_{3}\right)_{A_{4}}^{1,3}=\left(I_{3}\right)_{A_{4}}^{0,4}=0$. Consequently the sum of three and four-way correlations is given by

$$
16 N_{4,8}^{A_{1} A_{2} A_{3}}=16\left(\left|\left(I_{3}\right)_{A_{4}}^{4,0}\right|^{2}+6\left|\left(I_{3}\right)_{A_{4}}^{2,2}\right|^{2}\right) .
$$

Using the result of Eq. (25) and the fact that $\tau_{A_{i}\left|A_{j}\right| A_{k}}(\rho)=\tau_{A_{1}\left|A_{2}\right| A_{3}}(\rho)$, we obtain

$$
\tau_{A_{i}\left|A_{j}\right| A_{k}}(\rho) \leq \frac{4\left|c\left(a^{2}-d^{2}\right)\right|}{\left(|a|^{2}+|d|^{2}+2|c|^{2}+1\right)^{2}}\left(\frac{|| c\left(a^{2}-d^{2}\right)|-|\left(a^{2}-c^{2}\right)\left(d^{2}-c^{2}\right)||}{\left|c\left(a^{2}-d^{2}\right)\right|+\left|\left(a^{2}-c^{2}\right)\left(d^{2}-c^{2}\right)\right|}\right) .
$$

Correct bound calculated in [29] for this class of states is $\frac{4\left|c\left(a^{2}-d^{2}\right)\right|}{\left(|a|^{2}+|d|^{2}+2|c|^{2}+1\right)^{2}}$.

\section{Class III}

Three tangle vanishes on reduced state obtained by tracing out qubit $A_{2}$ or $A_{4}$ from state

$$
\left|G_{a b}^{(3)}\right\rangle=a(|0000\rangle+|1111\rangle)+b(|0101\rangle+|1010\rangle)+|0011\rangle+|0110\rangle,
$$

because $N_{4,8}^{A_{1} A_{3} A_{4}}=N_{4,8}^{A_{1} A_{2} A_{3}}=2\left|I_{4,8}\right|$. On the other hand, if qubit $A_{3}$ is traced out then non-zero three-qubit invariants $\left(I_{3}\right)_{A_{3}}^{0,4}=\frac{-4 a b}{\left(2|a|^{2}+2|b|^{2}+2\right)^{2}}$ and $\left(I_{3}\right)_{A_{3}}^{2,2}=\frac{2\left(a^{2}-b^{2}\right)^{2}}{3\left(2|a|^{2}+2|b|^{2}+2\right)^{2}}$, determine the three tangle. Four qubit invariant $\left|I_{4,8}\right|=3\left|\left(I_{3}\right)_{A_{l}}^{2,2}\right|^{2}$ and pure state three-way correlation are found to be $16\left(N_{4,8}^{A_{1} A_{2} A_{4}}-2\left|I_{4,8}\right|\right)=16\left|\left(I_{3}\right)_{A_{3}}^{0,4}\right|^{2} \cdot \mathrm{Using}$ the result given in Eq. (28), upper bound on three tangle for the partition $A_{1} A_{2} A_{4}$ reads as

$$
\tau_{A_{1}\left|A_{2}\right| A_{4}}(\rho) \leq \frac{4|a b|}{\left(|a|^{2}+|b|^{2}+1\right)^{2}} \frac{|4| a b|-| a^{2}-\left.b^{2}\right|^{2} \mid}{\left(4|a b|+\left|a^{2}-b^{2}\right|^{2}\right)} .
$$

Upper bound calculated in ref. [28] is $\tau_{A_{1}\left|A_{2}\right| A_{4}}(\rho) \leq \frac{4|a b|}{\left(|a|^{2}+|b|^{2}+1\right)^{2}}$. Our upper bound may also be compared with the convex roof for the same state reported in [26] to be

$$
\left[\tau_{A_{1}\left|A_{2}\right| A_{4}}(\rho)\right]^{\frac{1}{2}}=\max \left(0,\left(\frac{2 \sqrt{|a b|}}{\left(|a|^{2}+|b|^{2}+1\right)}\right) \frac{\left(4|a b|-\left|a^{2}-b^{2}\right|^{2}\right)}{4|a b|}\right) .
$$

\section{Class IV}

For entanglement class represented by

$$
\begin{aligned}
\left|G_{a b}^{(4)}\right\rangle & =a(|0000\rangle+|1111\rangle)+\frac{a+b}{2}(|1010\rangle+|0101\rangle) \\
& +\frac{a-b}{2}(|0110\rangle+|1001\rangle)+\frac{i}{\sqrt{2}}(|0010\rangle+|0001\rangle+|0111\rangle+|1011\rangle)
\end{aligned}
$$

all four reduced density matrices are found to have the same upper bound on three-tangle. Taking up the case of qubits $A_{1} A_{2} A_{3}$, only non-zero pure-state three-qubit invariant is $\left(I_{3}\right)_{A_{4}}^{0,4}$. Since $I_{4,8}=0$, three-tangle is equal to

$$
\tau_{A_{1}\left|A_{2}\right| A_{3}}(\rho)=\sqrt{N_{4,8}^{A_{1} A_{2} A_{3}}}=4\left|\left(I_{3}\right)_{A_{4}}^{0,4}\right|
$$

Substituting the value of $\left(I_{3}\right)_{A_{4}}^{0,4}$ in Eq. (41) and using $\tau_{A_{1}\left|A_{2}\right| A_{3}}(\rho)=\tau_{A_{i}\left|A_{j}\right| A_{k}}(\rho)$, we have the inequality

$$
\tau_{A_{i}\left|A_{j}\right| A_{k}}(\rho) \leq \frac{2\left|a^{2}-b^{2}\right|}{\left(2+3|a|^{2}+|b|^{2}\right)^{2}}
$$

which is the same as reported for this state in [28]. 


\section{E. Class V}

For representative of entanglement class V, which reads as

$$
\begin{aligned}
\left|G_{a}^{(5)}\right\rangle & =a(|0000\rangle+|1111\rangle+|0101\rangle+|1010\rangle) \\
& +i|0001\rangle+|0110\rangle-i|1011\rangle,
\end{aligned}
$$

non-zero three-qubit invariants of interest are

$$
\left(I_{3}\right)_{A_{4}}^{0,4}=\left(I_{3}\right)_{A_{2}}^{4,0}=\frac{-4 a^{2}}{\left(3+4|a|^{2}\right)^{2}},
$$

and

$$
\left(I_{3}\right)_{A_{3}}^{1,3}=\frac{-2 i a^{2}}{\left(3+4|a|^{2}\right)^{2}} ; \quad\left(I_{3}\right)_{A_{3}}^{0,4}=\frac{-1}{\left(3+4|a|^{2}\right)^{2}} .
$$

Consequently, $\tau_{A_{1}\left|A_{2}\right| A_{3}}(\rho)=\tau_{A_{1}\left|A_{3}\right| A_{4}}(\rho)$, such that

$$
\tau_{A_{1}\left|A_{2}\right| A_{3}}(\rho) \leq \frac{16\left|a^{2}\right|}{\left(3+4|a|^{2}\right)^{2}} .
$$

This bound coincides with the results from ref. [26] and ref. [28].

For the marginal state obtained by tracing out qubit $A_{3}$, the values of $\left(I_{3}\right)_{A_{3}}^{0,4}$ and $\left(I_{3}\right)_{A_{3}}^{1,3}$ are substituted in Eq. (31) to obtain

$$
\tau_{A_{1}\left|A_{2}\right| A_{4}}(\rho) \leq \frac{4}{\left(3+4|a|^{2}\right)^{2}}\left(\frac{1}{1+64|a|^{4}}\right) .
$$

In comparison, upper bound on three tangle calculated by Osterloh (Eq. 37 of ref. ([26])) corresponds to

$$
\tau_{A_{1}\left|A_{2}\right| A_{4}}(\rho) \leq \frac{4}{\left(3+4 a^{2}\right)^{2}}\left(\frac{1+64|a|^{2}}{\left(1+64|a|^{4}\right)^{2}}\right) .
$$

\section{F. Classes VI, VII, VIII and IX}

Non-zero pure state three-qubit invariants for class six state

$$
\left|G_{a}^{(6)}\right\rangle=a(|0000\rangle+|1111\rangle)+|0011\rangle+|0101\rangle+|0110\rangle,
$$

are given by $\left(I_{3}\right)_{A_{4}}^{2,2}=\left(I_{3}\right)_{A_{3}}^{2,2}=\left(I_{3}\right)_{A_{2}}^{2,2}=\frac{a^{4}}{6\left(3+2 a^{2}\right)^{2}}$. Consequently, $\tau_{A_{i}\left|A_{j}\right| A_{k}}(\rho)$ is zero on states of the entanglement type represented by state $\left|G_{a}^{(6)}\right\rangle$. States represented by

$$
\left|G^{(7)}\right\rangle=|0000\rangle+|0101\rangle+|1000\rangle+|1110\rangle
$$

and

$$
\left|G_{a b}^{(8)}\right\rangle=|0000\rangle+|1011\rangle+|1101\rangle+|1110\rangle
$$

differ in the amount of two-way correlations. For both the states, $\tau_{A_{1}\left|A_{j}\right| A_{k}}(\rho)=\frac{1}{4}, \tau_{A_{2}\left|A_{3}\right| A_{4}}(\rho)=0$, while state nine which reads as

$$
\left|G_{a b}^{(9)}\right\rangle=|0000\rangle+|0111\rangle
$$

has obviously $\tau_{A_{2}\left|A_{3}\right| A_{4}}(\rho)=\frac{1}{4}$. 


\section{UPPER BOUND ON THREE TANGLE AND OPTIMAL DECOMPOSITION OF A RANK TWO MIXED STATE}

The procedure of section III may be used as an additional tool to find the optimal decomposition $\left\{p_{i},\left|\phi_{i}\right\rangle\right\}$ that realizes the minimum in the definition (Eq. (3)) of three tangle of a mixed three-qubit state $\rho_{3}=\sum_{i} p_{i}\left|\phi_{i}\right\rangle\left\langle\phi_{i}\right|$. To write down the equations corresponding to Eq. (13) and Eq. (14), one calculates relevant three-qubit invariants of the purification of the state. Minimization may require solving a quartic equation. If an analytical solution is not available, then the roots of the equation may be found numerically. Consider a mixture of three-qubit pure states,

$$
\rho_{3}=p\left|\phi^{(0)}\right\rangle\left\langle\phi^{(0)}|+(1-p)| \phi^{(1)}\right\rangle\left\langle\phi^{(1)}\right|,
$$

where $\left\langle\phi_{1} \mid \phi_{0}\right\rangle=0$. Purification of the state can be written as

$$
\left|\Psi_{4}\right\rangle=\sqrt{p}\left|\phi^{(0)}\right\rangle|0\rangle+\exp (i \theta) \sqrt{1-p}\left|\phi^{(1)}\right\rangle|1\rangle .
$$

Action of $U(x)=\frac{1}{\sqrt{1+|x|^{2}}}\left[\begin{array}{cc}1 & -x^{*} \\ x & 1\end{array}\right]$, on fourth qubit of $\left|\Psi_{4}\right\rangle$ leads to state

$$
\left|\Psi_{4}(x)\right\rangle=\sqrt{p^{(0)}(x)}\left|\phi^{(0)}(x)\right\rangle|0\rangle+\sqrt{p^{(1)}(x)}\left|\phi^{(1)}(x)\right\rangle|1\rangle
$$

where

$$
\left|\phi^{(0)}(x)\right\rangle=\frac{\sqrt{p}\left|\phi^{(0)}\right\rangle-x^{*} \exp (i \theta) \sqrt{1-p}\left|\phi^{(1)}\right\rangle}{\sqrt{p+|x|^{2}(1-p)}} ; p^{(0)}(x)=\frac{p+|x|^{2}(1-p)}{1+|x|^{2}},
$$

and

$$
\left|\phi^{(1)}(x)\right\rangle=\frac{\exp (i \theta) \sqrt{1-p}\left|\phi^{(1)}\right\rangle+x \sqrt{p}\left|\phi^{(0)}\right\rangle}{\sqrt{p|x|^{2}+(1-p)}} ; p^{(1)}(x)=1-p^{(0)}(x) .
$$

The upper bound on three tangle of reduced state

$$
\rho(x)=\sum_{i=0}^{1} p^{(i)}(x)\left|\phi^{(i)}(x)\right\rangle\left\langle\phi^{(i)}(x)\right|,
$$

subject to the condition that $\rho(x)=\rho_{3}$, can be found by using the procedure outlined in section II. However, proper analysis of three tangles $\tau_{A_{1}\left|A_{2}\right| A_{3}}\left(\left|\phi^{(0)}(x)\right\rangle\right)$ and $\tau_{A_{1}\left|A_{2}\right| A_{3}}\left(\left|\phi^{(1)}(x)\right\rangle\right)$ along with the respective vectors, further aids in improving on the upper bound.

To illustrate, we recover the results for a mixed state studied in ref. [21], which reads as

$$
\rho_{3}=p|G H Z\rangle\langle G H Z|+(1-p)| W\rangle\langle W|,
$$

such that $|G H Z\rangle=\frac{1}{\sqrt{2}}(|000\rangle+|111\rangle)$ and $|W\rangle=\frac{1}{\sqrt{3}}(|100\rangle+|010\rangle+|001\rangle)$. Values of relevant three-qubit invariants for the purification

$$
|\Psi\rangle=\sqrt{p}|G H Z\rangle|0\rangle+\exp (i \theta) \sqrt{(1-p)}|W\rangle|0\rangle
$$

are $\left(I_{3}\right)_{A_{4}}^{4,0}=\frac{p^{2}}{4}$ and $4\left(I_{3}\right)_{A_{4}}^{1,3}=4 \frac{\exp (i 3 \theta) \sqrt{p(1-p)^{3}}}{3 \sqrt{6}}$. State $\left|\Psi_{4}(x)\right\rangle$ (Eq. (54) )obtained after a unitary transformation $U(x)$ on fourth qubit contains normalized three-qubit states

$$
\left|\phi^{(0)}(x, \theta)\right\rangle=\frac{\sqrt{p}|G H Z\rangle-x^{*} \exp (i \theta) \sqrt{1-p}|W\rangle}{\sqrt{p+|x|^{2}(1-p)}}, \quad p^{(0)}(x)=\frac{p+|x|^{2}(1-p)}{1+|x|^{2}},
$$

and

$$
\left|\phi^{(1)}(x, \theta)\right\rangle=\frac{\exp (i \theta) \sqrt{1-p}|W\rangle+x \sqrt{p}|G H Z\rangle}{\sqrt{(1-p)+p|x|^{2}}} ; \quad p^{(1)}(x)=1-p^{(0)}(x),
$$


such that $x$ dependent three tangle is given by

$$
\left[\tau_{A_{1}\left|A_{2}\right| A_{3}}(\rho(x))\right]^{\frac{1}{2}}=\sum_{i=1,2} p^{(i)}(x)\left[\tau_{A_{1}\left|A_{2}\right| A_{3}}\left(\left|\phi^{(i)}(x, \theta)\right\rangle\right)\right]^{\frac{1}{2}} .
$$

Three tangles of vectors in the superposition are

$$
\tau_{A_{1}\left|A_{2}\right| A_{3}}\left(\left|\phi^{(0)}(x, \theta)\right\rangle\right)=\frac{4\left|\left(I_{3}\right)_{A_{4}}^{4,0}-4\left(x^{*}\right)^{3}\left(I_{3}\right)_{A_{4}}^{1,3}\right|}{\left(p+(1-p)|x|^{2}\right)^{2}},
$$

and

$$
\tau_{A_{1}\left|A_{2}\right| A_{3}}\left(\left|\phi^{(1)}(x, \theta)\right\rangle\right)=\frac{4\left|x^{4}\left(I_{3}\right)_{A_{4}}^{4,0}+4 x\left(I_{3}\right)_{A_{4}}^{1,3}\right|}{\left(|x|^{2} p+(1-p)\right)^{2}} .
$$

Substituting the values of $\left(I_{3}\right)_{A_{4}}^{4,0}$ and $4\left(I_{3}\right)_{A_{4}}^{1,3}$ in $\tau_{A_{1}\left|A_{2}\right| A_{3}}\left(\left|\phi^{(0)}(x, \theta)\right\rangle\right)$, we obtain

$$
\tau_{A_{1}\left|A_{2}\right| A_{3}}\left(\left|\phi^{(0)}(x, \theta)\right\rangle\right)=\frac{4\left|\frac{p^{2}}{4}-4\left(x^{*}\right)^{3} \frac{\exp (i 3 \theta) \sqrt{p(1-p)^{3}}}{3 \sqrt{6}}\right|}{\left(p+(1-p)|x|^{2}\right)^{2}}
$$

which is a periodic function of $\theta$ with a period of $2 \pi / 3$. For the choice $\theta_{n}=2 \pi n / 3, n=0,1$, 2, three-tangle $\tau_{A_{1}\left|A_{2}\right| A_{3}}\left(\left|\phi^{(0)}\left(x_{0}, \theta_{n}\right)\right\rangle\right)$ becomes zero for $x_{0}=\left(\frac{3 \times 2^{\frac{5}{3}} p}{16(1-p)}\right)^{\frac{1}{2}}$. A closer examination shows that for $p \leq 0.626851$ the value of $x_{0}$ lies within the range $0 \leq|x| \leq 1$, while for $p>0.626851, \tau_{A_{1}\left|A_{2}\right| A_{3}}\left(\left|\phi^{(0)}\left(x, \theta_{n}\right)\right\rangle\right)>0$ being minimum at $x=1$. For $0 \leq p \leq 0.626851$, three-tangle $\left[\tau_{A_{1}\left|A_{2}\right| A_{3}}\left(\rho_{3}\left(x_{0}\right)\right)\right]=0$, for the mixed state

$$
\rho_{3}\left(x_{0}\right)=\frac{1}{3} \sum_{n=0}^{2}\left|\phi^{(0)}\left(x_{0}, \theta_{n}\right)\right\rangle\left\langle\phi^{(0)}\left(x_{0}, \theta_{n}\right)\right|,
$$

where

$$
\left|\phi^{(0)}\left(x_{0}, \theta_{n}\right)\right\rangle=\frac{4|G H Z\rangle-\exp \left(i \theta_{n}\right) \sqrt{3 \times 2^{\frac{5}{3}}}|W\rangle}{4 \sqrt{\left(1+\frac{3}{8} 2^{\frac{2}{3}}\right)}} .
$$

Hence the decomposition of $\rho_{3}$ of Eq. (52), with $\left[\tau_{A_{1}\left|A_{2}\right| A_{3}}\left(\rho_{3}\left(x_{0}\right)\right)\right]=0$ can be written as

$$
\rho_{3}=p\left(1+\frac{3}{8} 2^{\frac{2}{3}}\right) \rho_{3}\left(x_{0}\right)+\left(1-p\left(1+\frac{3}{8} 2^{\frac{2}{3}}\right)\right)|W\rangle\langle W| .
$$

Since for $p>0.626851$, vectors $\left|\phi^{(0)}\left(1, \theta_{n}\right)\right\rangle$ have lowest value of three-tangle the upper bound on three-tangle is given by

$$
\tau_{A_{1}\left|A_{2}\right| A_{3}}\left(\rho_{3}(1)\right)=\left|\frac{p^{2}}{4}-\frac{4 \sqrt{p(1-p)^{3}}}{3 \sqrt{6}}\right|,
$$

and the corresponding decomposition is

$$
\rho_{3}=\frac{1}{3} \sum_{n=0}^{2}\left|\phi^{(0)}\left(1, \theta_{n}\right)\right\rangle\left\langle\phi^{(0)}\left(1, \theta_{n}\right)\right| .
$$

Similar arguments may be used to find upper bound on three-tangle of an arbitrary rank-two mixed state of three qubits. 


\section{CONCLUDING REMARKS}

For most of the four-qubit states, our bound on three-tangle of reduced state is tighter than that used in ref. [28]. A careful examination shows that the upper bounds on three tangles listed in Table I of ref. [28] are given by $4 \sqrt{N_{4,8}^{A_{i} A_{j} A_{k}}-\left|2 I_{4,8}\right|}$. On the other hand for states that correspond to cases (vi), (v), and (vi) of section III, three tangle satisfies

$$
\tau_{A_{i}\left|A_{j}\right| A_{k}}(\rho) \leq 4 F \sqrt{N_{4,8}^{A_{i} A_{j} A_{k}}-\left|2 I_{4,8}\right|}, \quad F \leq 1
$$

In ref. [26] unitary on three qubit states is used to obtain minimal characteristic curves to construct convex roof of three tangle for nine classes of four-qubit states. Comparing the upper bound obtained by unitary on fourth qubit with results for tangle corresponding to minimal characteristic curve in ref. [26] it is noted that for states in class II, class III, and class V our value is lower for certain ranges of state parameters than that of ref. [26]. For all other cases, the result obtained is the same.

Correct understanding of relation between pure state correlations and entanglement of marginal states is crucial to discovering the form of monogamy relations for multipartite entanglement. After examining the upper bounds for nine classes of four-qubit states $|\Psi\rangle$, we conclude that

$$
\begin{aligned}
& 4\left|\left(I_{3}\right)_{A_{4}}^{4,0}\right|+4\left|\left(I_{3}\right)_{A_{4}}^{0,4}\right| \geq \tau_{A_{1}\left|A_{2}\right| A_{3}}^{u p}\left(\rho_{123}\right) \geq \tau_{A_{1}\left|A_{2}\right| A_{3}}\left(\rho_{123}\right) \\
& 4\left|\left(I_{3}\right)_{A_{3}}^{4,0}\right|+4\left|\left(I_{3}\right)_{A_{3}}^{0,4}\right| \geq \tau_{A_{1}\left|A_{2}\right| A_{4}}^{u p}\left(\rho_{124}\right) \geq \tau_{A_{1}\left|A_{2}\right| A_{4}}\left(\rho_{124}\right)
\end{aligned}
$$

and

$$
4\left|\left(I_{3}\right)_{A_{2}}^{4,0}\right|+4\left|\left(I_{3}\right)_{A_{2}}^{0,4}\right| \geq \tau_{A_{1}\left|A_{3}\right| A_{4}}^{u p}\left(\rho_{134}\right) \geq \tau_{A_{1}\left|A_{3}\right| A_{4}}\left(\rho_{134}\right)
$$

This result is used in 31] to analytically write down the correct monogamy inequality for four-qubit states.

To conclude, the method outlined in this letter can be used to obtain three tangle of a rank-two three-qubit mixed state. Three-tangles are of interest to establish the connection between condensed-matter physics and quantum information [32] as well as to better understand the connection between quantum correlations in spin systems undergoing quantum phase transitions [33].

Financial support from Universidade Estadual de Londrina PR, Brazil is acknowledged.

\section{Appendix A: Degree four three qubit invariants of four-qubit state}

Degree four three-qubit invariants of four-qubit state relevant to constructing the upper bound on $\tau_{A_{1}\left|A_{2}\right| A_{3}}(\rho)$ in terms of two-qubit invariants for the pair $A_{1} A_{2}$ are listed below:

$$
\begin{aligned}
\left(I_{3}\right)_{A_{4}}^{4,0}=\left(D_{\left(A_{4}\right)_{0}}^{000}+D_{\left(A_{4}\right)_{0}}^{001}\right)^{2}-4 D_{\left(A_{3}\right)_{1}\left(A_{4}\right)_{0}}^{00} D_{\left(A_{3}\right)_{0}\left(A_{4}\right)_{0}}^{00} \\
\left(I_{3}\right)_{A_{4}}^{3,1}=\frac{1}{2}\left(D_{\left(A_{4}\right)_{0}}^{000}+D_{\left(A_{4}\right)_{0}}^{001}\right)\left(D^{0000}+D^{0001}+D^{0010}+D^{0011}\right) \\
-\left[D_{\left(A_{3}\right)_{1}\left(A_{4}\right)_{0}}^{00}\left(D_{\left(A_{3}\right)_{0}}^{000}+D_{\left(A_{3}\right)_{0}}^{001}\right)+D_{\left(A_{3}\right)_{0}\left(A_{4}\right)_{0}}^{00}\left(D_{\left(A_{3}\right)_{1}}^{000}+D_{\left.\left(A_{3}\right)_{1}\right)}^{001}\right)\right] \\
\left(I_{3}\right)_{A_{4}}^{2,2}=\frac{1}{6}\left(D^{0000}+D^{0001}+D^{0010}+D^{0011}\right)^{2} \\
-\frac{2}{3}\left(D_{\left(A_{3}\right)_{1}}^{000}+D_{\left(A_{3}\right)_{1}}^{001}\right)\left(D_{\left(A_{3}\right)_{0}}^{000}+D_{\left(A_{3}\right)_{0}}^{001}\right) \\
+\frac{1}{3}\left(D_{\left(A_{4}\right)_{0}}^{000}+D_{\left(A_{4}\right)_{0}}^{001}\right)\left(D_{\left(A_{4}\right)_{1}}^{000}+D_{\left(A_{4}\right)_{1}}^{001}\right) \\
-\frac{2}{3}\left(D_{\left(A_{3}\right)_{1}\left(A_{4}\right)_{0}}^{000} D_{\left(A_{3}\right)_{0}\left(A_{4}\right)_{1}}^{00}+D_{\left(A_{3}\right)_{0}\left(A_{4}\right)_{0}}^{00} D_{\left(A_{3}\right)_{1}\left(A_{4}\right)_{1}}^{00}\right)
\end{aligned}
$$




$$
\begin{aligned}
\left(I_{3}\right)_{A_{4}}^{1,3}= & \frac{1}{2}\left(D^{0000}+D^{0001}+D^{0010}+D^{0011}\right)\left(D_{\left(A_{4}\right)_{1}}^{000}+D_{\left(A_{4}\right)_{1}}^{001}\right) \\
& -\left[D_{\left(A_{3}\right)_{1}\left(A_{4}\right)_{1}}^{00}\left(D_{\left(A_{3}\right)_{0}}^{000}+D_{\left(A_{3}\right)_{0}}^{001}\right)+\left(D_{\left(A_{3}\right)_{1}}^{000}+D_{\left(A_{3}\right)_{1}}^{001}\right) D_{\left(A_{3}\right)_{0}\left(A_{4}\right)_{1}}^{00}\right]
\end{aligned}
$$

and

$$
\left(I_{3}\right)_{A_{4}}^{0,4}=\left(D_{\left(A_{4}\right)_{1}}^{000}+D_{\left(A_{4}\right)_{1}}^{001}\right)^{2}-4 D_{\left(A_{3}\right)_{1}\left(A_{4}\right)_{1}}^{00} D_{\left(A_{3}\right)_{0}\left(A_{4}\right)_{1}}^{00}
$$

[1] R. Horodecki, P. Horodecki, M. Horodecki, and K. Horodecki, Rev. Mod. Phys. 81, 865 (2009); O. Gühne and G. Tóth, Phys. Rep. 474, 1 (2009).

[2] V. Dodonov and V. Man'ko, Theory of Nonclassical States of Light (Taylor \& Francis, New York, 2003).

[3] J. Perina, Z. Hradil, and B. Jurco, Quantum Optics and Fundamentals of Physics (Kluwer, Dordrecht, 1994).

[4] M. A. Nielsen and I. L. Chuang, Quantum Computation and Quantum Information (Cambridge University Press, Cambridge, UK, 2000).

[5] N. Lambert, Y. N. Chen, Y. C. Chen, C. M. Li, G. Y. Chen, and F. Nori, Quantum biology, Nat. Phys. 9, 10 (2013).

[6] C. M. Li, N. Lambert, Y. N. Chen, G. Y. Chen, and F. Nori, Witnessing quantum coherence: From solid-state to biological systems, Sci. Rep. 2, 885 (2012).

[7] O. Gühne, M. Reimpell, and R. F. Werner, Phys. Rev. Lett. 98, 110502 (2007); Phys. Rev. A 77, 052317 (2008).

[8] J. Eisert, F. G. S. L. Brandao, and K. M. R. Audenaert, New J. Phys. 9, 46 (2007).

[9] H. Wunderlich and M. B. Plenio, J. Mod. Opt. 56, 2100 (2009).

[10] S. Sahling, G. Remenyi, C. Paulsen, P. Monceau, V. Saligrama, C. Marin, A. Revcolevschi, L. P. Regnault, S. Raymond and J. E. Lorenzo, Nature Physics 11, 255-260 (2015).

[11] A. Osterloh, L. Amico, G. Falci, and R. Fazio, Nature (London) 416, 608 (2002); M. Cramer, A. Bernard, N. Fabbri, L. Fallani, C. Fort, S. Rosi, F. Caruso, M. Inguscio, and M. B. Plenio, Nat. Commun. 4, 2161 (2013).

[12] K. Zyczkowski, P. Horodecki, A. Sanpera, and M. Lewenstein, Phys. Rev. A 58, 883 (1998).

[13] G. Vidal and R. F. Werner, Phys. Rev. A Vol. 65, 032314 (2002).

[14] S. Hill and W. K. Wootters, Phys. Rev. Lett. 78 (1997) 5022.

[15] V. Coffman, J. Kundu, and W. K. Wootters, Phys. Rev. A 61, 052306 (2000).

[16] S. S. Sharma and N. K. Sharma, Phys. Rev. A 87, 022335 (2013).

[17] M. Grassl, M. Rotteler and T. Beth, Phys. Rev. A 58, 1833 (1998).

[18] E. Briand, J.-Gabriel. Luque and J.-Y. Thibon, J. Phys. A: Math. Gen. 36, 9915 (2003).

[19] J.-G. Luque and J.-Y. Thibon, J. Phys. A 39, 371 (2006).

[20] Sharma, S.S. and Sharma, N.K. Quantum Inf Process, Vol. 15, 4973-4993 (2016).

[21] R. Lohmayer, A. Osterloh, J. Siewert, and A. Uhlmann, Phys. Rev. Lett. 97, 260502 (2006).

[22] C. Eltschka, A. Osterloh, J. Siewert, and A. Uhlmann, New J. Phys. 10, 043014 (2008).

[23] C. Eltschka and J. Siewert, Phys. Rev. Lett. 108, 020502 (2012).

[24] C. Eltschka and J. Siewert, Phys. Rev. A 89, 022312 (2014).

[25] G. Tóth, T. Moroder, and O. Gühne, PRL 114, 160501 (2015).

[26] A. Osterloh, Phys. Rev. A 94, 012323(2016).

[27] F. Verstraete, J. Dehaene, B. De Moor, and H. Verschelde, Phys. Rev. A 65, 052112 (2002).

[28] B. Regula, S. Di Martino, S. Lee, and G. Adesso, PRL 113, 110501 (2014).

[29] B. Regula, S. Di Martino, S. Lee, and G. Adesso, PRL 116, 049902(E) (2016).

[30] S. Rodriques, N. Datta, and P. J. Love, Phys. Rev. A 90, 012340 (2014).

[31] On monogamy of four qubit entanglement, S. Shelly Sharma and N. K. Sharma, arXiv:1703.09861 [quant-ph](March 2017).

[32] L. Amico, R. Fazio, A. Osterloh, and V. Vedral, Rev. Mod. Phys. 80, 517 (2008).

[33] Werlang, T., Trippe, C., Ribeiro, G. A. P. \& Rigolin, G. Phys. Rev. Lett. 105, 095702 (2010). 